\title{
A practical and robust method to compute the boundary of three-dimensional axis-aligned boxes
}

\author{
Daniel López Monterde, Jonàs Martínez, Marc Vigo and Núria Pla \\ Departament de Llenguatges i Sistemes Informàtics, Universitat Politècnica de Catalunya, Edifici ETSEIB, Diagonal 647, \\ 8 a Planta E, 08028 Barcelona, Spain \\ \{dlopez, jmartinez, marc, nuria\}@lsi.upc.edu
}

Keywords: boundary representation, union of boxes, orthogonal polyhedra

\begin{abstract}
The union of axis-aligned boxes results in a constrained structure that is advantageous for solving certain geometrical problems. A widely used scheme for solid modelling systems is the boundary representation (Brep). We present a method to obtain the B-rep of a union of axis-aligned boxes. Our method computes all boundary vertices, and additional information for each vertex that allows us to apply already existing methods to extract the B-rep. It is based on dividing the three-dimensional problem into two-dimensional boundary computations and combining their results. The method can deal with all geometrical degeneracies that may arise. Experimental results prove that our approach outperforms existing general methods, both in efficiency and robustness.
\end{abstract}

\section{INTRODUCTION}

Many of the current solid modelling systems are based on Boundary Representations (B-rep) and Constructive Solid Geometry (CSG). Computing the Brep of the resulting solid of CSG, after performing boolean operations, is a fundamental operation in these systems. The problem of robust and accurate computation of the boundary is considered one of the difficult problems in solid modelling (Hoffmann, 1996). Most of the effort in the subject is focused on boolean operations between polyhedral or algebraic objects (Requicha and Voelcker, 1985; Thibault and Naylor, 1987). The restricted case of threedimensional axis-aligned boxes has received less interest, even though its bounded structure can lead to robust and simple algorithms. Furthermore, the union of axis-aligned boxes is an orthogonal polyhedron. Their constrained structure has enabled advances on complex or unsolved problems for arbitrary shapes (Bournez et al., 1999; Vigo et al., 2012).

Orthogonal polyhedra have been directly studied for mesh reconstruction from scanner data (Biedl et al., 2009) and urban modelling (Sugihara and Hayashi, 2008). They have also been used to bound or approximate more complex shapes, with applications in spatial databases (Esperança and Samet, 1997), and motion planning (Albers et al., 1999). In particular, an efficient algorithm that obtains the union of boxes is required to compute the skeleton of orthogonal polyhedra (Martinez et al., 2013)

The problem of computing the two-dimensional boundary of the union of a set of axis-aligned rectangles, among other properties, sparked interest in the 1980s (Jr. and Preparata, 1980; Wood, 1984; Güting, 1984). Several methods were proposed, and the problem has been optimally solved since then. Its extension to three dimensions, however, has received little attention in the literature.

Our aim is to fill that gap, by presenting an algorithm that computes the boundary of the union of a set of axis-aligned three-dimensional boxes. The output of the presented method is a B-rep of the union of the boxes. For this purpose, we apply the results presented by Vigo et al. (Vigo et al., 2012). Their algorithm obtains the B-rep from the list of vertices in an orthogonal polyhedron's boundary and additional information concerning the local topology of each vertex. Our computes this information in order to apply Vigo et al.'s algorithm and obtain a B-rep.

Our approach consists in dividing the overall computation into a series of 2D boundary computations, and then combining their results to obtain the 3D boundary. The obtained results demonstrate that the robustness of the 2D computation, which is much easier to obtain, directly implies the robustness in the three-dimensional case. Our method makes use of an axis-aligned plane, which is swept through the set of 
boxes. The plane is positioned between all pairs of consecutive faces, its intersection with the boxes inducing a set of rectangles. The $2 \mathrm{D}$ boundary of the set of rectangles is computed using already existing methods. Then, the final 3D boundary is obtained by comparing consecutive 2D boundaries.

One of the most challenging aspects of geometrical algorithms is to achieve robustness, given the variety of possible inputs (Hoffmann, 1989). Even when the domain is restricted to axis-aligned polyhedra, degenerate cases can still pose a problem. A geometrical degeneracy can be caused by the existance of coplanar faces, colinear edges, or coincident vertices. Nontrihedral vertices can also be considered degeneracies, and they can be originated from the previously mentioned degeneracy kinds.

Our method is robust to all cases, and it generates the expected output for any given input. It is able to handle floating point numbers, and, most importantly, generates no underflow conditions, by applying only comparison and assignation operators on them.

Besides its robustness, our algorithm has other noteworthy positive properties. Additionally, it requires no complex data structures to be implemented. Due to separating 3D from 2D concerns, the method is highly parallelizable.

\section{RELATED WORK}

The properties of union hyper-rectangles have been the focus of theoretical study. It is known that the union of $n$ axis-aligned boxes in dimension $d$ has a worst-case combinatorial complexity of $\theta\left(n^{d}\right)$. If the boxes are hypercubes, the union has combinatorial complexity of $O\left(n^{\lceil d / 2\rceil}\right)$. If the hypercubes have constant size the complexity reduces to $O\left(n^{\lfloor d / 2\rfloor}\right)$ (Boissonnat et al., 1998). Kaplan et al. presented an algorithm to compute the union of $d$-dimensional hypercubes of constant size in $O\left(n^{\lfloor d / 2\rfloor}\right.$ polylog $\left.n\right)$ time (Kaplan et al., 2007).

In the literature, most of the effort is devoted to compute the volume of the union, which is known as Klee's measure problem (Klee, 1977), rather than explicitly constructing the union. Interestingly, when $d>2$ the volume can be computed more efficiently than the union. For example, the algorithm of Overmars and Yap runs in $O\left(n^{d / 2} \log n\right)$ time (Overmars and Yap, 1991). It remains an open question whether faster algorithms are possible, or if it is possible to prove tighter lower bounds. In particular, it remains open whether the algorithm's running time must depend on d (Chan, 2010). Unfortunately, as the worstcase complexity of the union of axis aligned boxes is $\theta\left(n^{d}\right)$, theoretical efforts have been focused on the efficient computation of their volume.

Two different main strategies were proposed to compute the union of two-dimensional axis-aligned rectangles: sweep line (Jr. and Preparata, 1980) and divide and conquer (Güting, 1984) algorithms. Both algorithm types run in $O(n \log n+k)$ time, where $k$ is the number of vertices of the union.

Concerning the three-dimensional case, there exist several classical methods to compute the boundary of CSG (Requicha and Voelcker, 1985). Most of them classify the set of CSG primitives with some kind of spatial decomposition scheme (Thibault and Naylor, 1987). Hachenberger et al. have studied and optimized boolean operations on Nef Polyhedra, and that handle all cases, including all degeneracies (Hachenberger and Kettner, 2005). Baumann et al. compute the union of three dimensional axis-aligned boxes, represented by a set of enclosing rectangles, by an octree-based incremental algorithm (Baumann et al., 2008). Campen et al. presented a robust method that computes boolean operations using an adaptive octree with nested binary space partitions (Campen and Kobbelt, 2010). Schifko et al. presented a robust sweeping plane algorithm to compute boolean operations on meshes, based on R-trees (Schifko et al., 2010).

\section{PREVIOUS DEFINITIONS}

In this section we introduce some basic definitions used throghout the paper.

A polygon is a bounded subset of $\mathbb{R}^{2}$ enclosed by a finite set of line segments, called edges. The edges meet only at their endpoints, called vertices. A polygon face is a single connected component of a polygon.

An orthogonal polygon is a polygon enclosed by axis-aligned edges. An edge is called vertical if it is parallel to the ordinate and horizontal if it is parallel to the abscissa.

An orthogonal manifold polygon is an orthogonal polygon such that every vertex is shared by a vertical and a horizontal edge.

An orthogonal pseudomanifold polygon is a generalization of an orthogonal manifold polygon, which allows non-manifold vertices. A non-manifold vertex is a vertex polygon with four incident edges.

A polyhedron is a bounded subset of $\mathbb{R}^{3}$ enclosed by a finite set of non-intersecting planar polygons, called faces. 

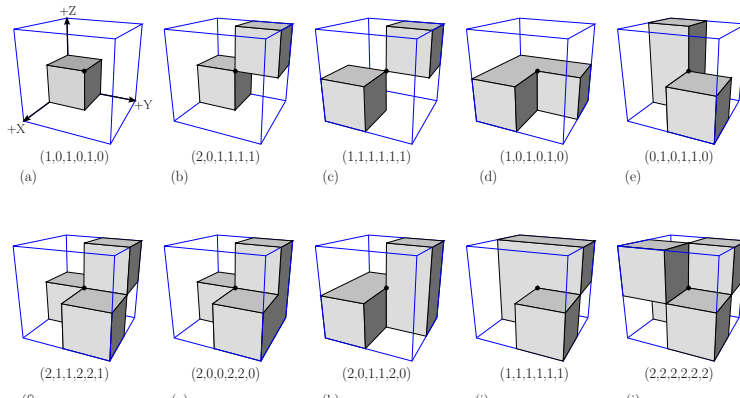

(h) (j)

Figure 1: Possible vertex configurations of an orthogonal pseudomanifold polyhedron. The six numbers below each configuration correspond to the face degrees that must be generated for them $(+x,-x,+y,-y,+z,-z)$

A trihedral vertex is a vertex of a polyhedron that has exactly three incident faces.

An orthogonal polyhedron is a polyhedron enclosed by axis-aligned faces.

An orthogonal manifold polyhedron is an orthogonal polyhedron such that every edge of a face is shared by exactly one other face.

An orthogonal pseudomanifold polyhedron is a generalization of an orthogonal manifold polyhedron, which allows non-manifold edges or vertices. A non-manifold edge is an edge adjacent to four faces and a non-manifold vertex is a non-trihedral vertex.

A boundary representation model (B-rep) represents a shape by a collection of its boundary elements and their topological relationships. Boundary representation models are composed of two parts: geometry (surfaces, curves and points) and topology. The main topological items are faces, edges and vertices. A face is a bounded portion of a surface; an edge is a bounded piece of a curve and a vertex lies at a point. The topological information defines the relationship among the geometric elements.

\section{VERTEX CONFIGURATIONS}

In this section, we define the additional information that needs to be computed for every boundary vertex. This information encodes the local topology of the vertex (see Figure 1) and it is required to prevent ambiguity in the reconstruction. It is the input needed, in addition to the vertex coordinates, for the B-rep extraction by Vigo et al. (Vigo et al., 2012). There are ten basic local configurations for a vertex. All the other possible configurations can be derived from

these by considering only rotation and complement operations (Aguilera, 1998).

Each possible configuration requires specific information to be computed (see Figure 1). This corresponds to the number of different faces converging at the vertex that are oriented in each of the six orthogonal directions $(+x,-x,+y,-y,+z,-z)$. For this reason, we refer to this information as vertex face degrees. There is one special case, (h), in which exactly four different faces intersect at the vertex. This configuration requires an exception to be made to the general rule. In this case, for the B-rep reconstruction to work, the two non-coplanar faces intersecting at the vertex have to be counted twice.

Our approach for computing the vertex face degrees is based on the following observations:

- Trihedral vertices, such as the one in $(a)$, have a total face degree of three, one for each axis.

- The rest of the vertex configurations can be obtained by considering them as a combination of a number of trihedral vertices.

Cases $(d)$ and $(e)$, as well as $(a)$, can be obtained with a single trihedral vertex. Cases $(b, c, g, h, i)$ can be obtained by combining the face degrees of two vertices. Case $(f)$ needs the combination of three vertices, and case $(j)$ needs to combine four trihedral vertices (see Figure 1).

These observations allow us to design our method so that it obtains only trihedral vertices. For the configurations where more than one vertex is needed to form the vertex face degrees, the method obtains each trihedral vertex independently. It is easily seen that it is possible to decompose all configurations into specific trihedral vertices, the degrees of which can be combined into the required ones. After all trihedral vertices are computed, those sharing the same coordinates are merged into a single vertex. That vertex has as its face degrees the sum of the face degrees of all the vertices that shared its coordinates.

\section{TWO-DIMENSIONAL BOUNDARY EXTRACTION}

We propose an optimal method to solve the twodimensional boundary problem. Since the implemented method differs from it, it is also detailed afterwards.

Input Set of axis-aligned rectangles

Output Set of vertices that form the boundary of the union of the input rectangles, and the $\mathrm{x}$ and $\mathrm{y}$ directions of each vertex 


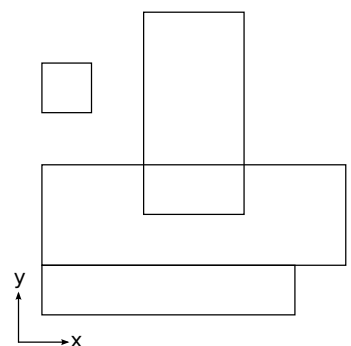

(a)

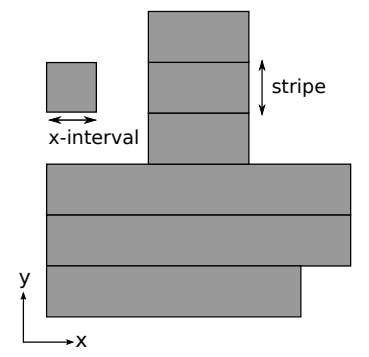

(b)
Figure 2: (a) Original set of rectangles. (b) Representation obtained after the first step.

\subsection{Optimal method}

Each 2D boundary is computed by the time-optimal method described by Güting (Güting, 1984). The method takes a set of axis aligned rectangles as its input, and obtains the list of all edges in the boundary of their union. However, it does not distinguish the edges that define external boundaries from those which define polygon holes. The method is divided in two steps. In the first step, it computes a representation of the union. In the second, it uses the obtained representation to compute the boundary edges. Because we require additional information for every vertex, we extend the second step of the algorithm.

The first step uses a divide and conquer approach. Each rectangle is represented by its two vertical edges: a technique Güting calls separational representation. The representation obtained (shown in Figure 2(b)) is a set of horizontal stripes spanning the polygon that constitutes the union. Each stripe contains a binary tree with the $x$-intervals where it is visible.

In the second step, the obtained representation is used to compute the boundary. Each horizontal edge in the input set of rectangles is considered. It is matched to one of its adjacent stripes, which is the stripe above it if the edge is top and the stripe below it if it is bottom. The tree of $x$-intervals associated with the stripe is queried with the $x$-interval corresponding to the edge. The leaves in the tree that are within the edge's interval represent boundaries of the polygon, and are therefore vertices of the boundary.

At this point, all horizontal edges have been computed, so we have all the vertices in the boundary.

\subsubsection{Vertex face degree computation}

We have to adapt the algorithm so that it generates the information needed for the B-rep extraction. Having already shown how the vertices of the boundary are obtained, we consider the problem of generating

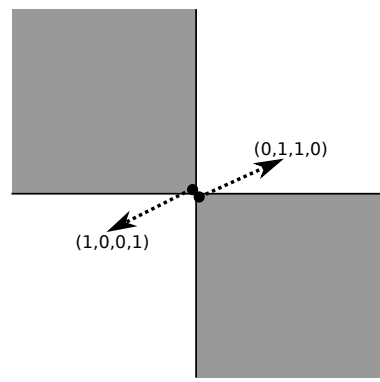

Figure 3: A non-manifold vertex in two dimensions, and its decomposition in two vertices. The numbers indicate the face degrees for each vertex, in $(+x,-x,+y,-y)$ format.

the face degrees for each of them. During the second step of the 2D computation, we obtain the vertex face degrees in the $x$ and $y$ axes.

The $x$ face degrees indicate whether the orientation of face that generated the vertex in this 2D boundary was right $(+x)$ or left $(-x)$. To compute them, we need to know what side of the $x$-interval the vertex was obtained from. If it was left end, the $-x$ degree of the vertex is 1 , and the $+x$ degree is 0 . And viceversa, $+x=1$ and $-x=0$, if it was the right end.

Analogously, the $y$ degrees indicate whether the face that generated the vertex was facing top $(+y)$ or bottom $(-y)$. They can be computed in a straightforward way. In this case, we need to know if the edge they were obtained from was a top or a bottom edge. For the top edges, we have 1 in the $+y$ degree and 0 in the $-y$; and viceversa for bottom edges.

Trying to apply the above principles to nonmanifold vertices leads us to ambiguity. This is due to the fact that non-manifold vertices in two dimensions are intersected by four segments (see Figure 3 ). These vertices appear when two different rectangles have opposite vertices in the same space coordinates. Our method for dealing with non-manifold vertices consists of considering them a combination of two independent vertices. This leaves us with two vertices in the same coordinates, pointing at opposed directions (see Figure 3). Each of them generates a trihedral vertex when extended to the third dimension, fulfilling the observations in Section 4. As we already mentioned, the resulting face degrees for that vertex are obtained from the sum of the trihedral vertices sharing the same coordinates.

\subsection{Actual implementation}

We implemented an algorithm inspired on the one proposed by Güting(Güting, 1984). Like Güting's, our algorithm is divided in two steps. In the first step, a new representation of the set of boxes is obtained, which consists of all the horizontal stripes and the ver- 
tical intervals of each of them (see Figure 2(a)). In the second step, that representation is used to compute the vertices that form the boundary. Güting proposes a divide and conquer method for the first step, which we already described in section 5.1 Instead, we use a sweep line version which generates the boundary in an incremental way.

Status data structure All the information of the boundary processed until the current point, in the form of a list of horizontal stripes.

Horizontal stripe A horizontal section of the boundary, divided by vertical $x$-intervals.

$x$-interval An x coordinate delimiting a vertical interval of horizontal stripe.

Edge Edge of a rectangle, with information about its opposite edge.

The first step uses a sweep line algorithm, where each edge is an event point. Each rectangle is separationally represented by its two y-edges. We start with an empty status data structure, and iteratively add to it one edge at a time. The status data structure keeps all the information of the boundary that has been processed up to the current event point, in the form of the list of all sections. Each new edge is compared to all the sections in the status data structure, and it corresponds to one of 4 cases:

1. If the edge does not overlap in any way a stripe, the stripe will not be modified.

2. If it completely overlaps a stripe, a new $x$-interval will be merged into the stripe.

3. If one of the edge's ends is in the middle of a stripe, the stripe will be split in 2 , and each side will be handled as one of the above cases.

4. If it covers an area where there was no stripe, a new stripe will be generated with the edge as a $x$-interval.

The stripes are compared with the edge from bottom to top. We use an example to clarify the method (see Figure 4). Stripe 1 doesn't overlap the edge (case 1) and is not changed. Stripe 2 is split in half by the edge (case 3 ), generating two stripes, $2 a$ and $2 b$, of which $2 \mathrm{a}$ is left unchanged, and $2 \mathrm{~b}$ has the new $x$-interval from the edge added to it. A new stripe, which we called 4, is created between 2 and 3 , since there was empty space (case 4). The edge is merged into stripe 3 (case 2), and stripe 5 is created (case 4) on the empty space over stripe 3 .

Each stripe retains the information of what length it covers, obtained from the edges that are added to it. This way, if an edge we're merging would be covered

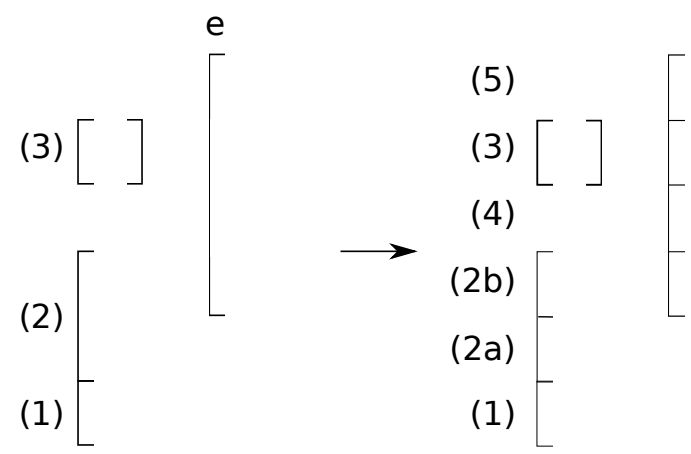

Figure 4: Visual representation of the merging process. The left side shows the status data structure halfway during processing (with stripes 1-3), and the edge $e$ that will be merged into it. The right side shows the status data structure after edge $e$ was merged.

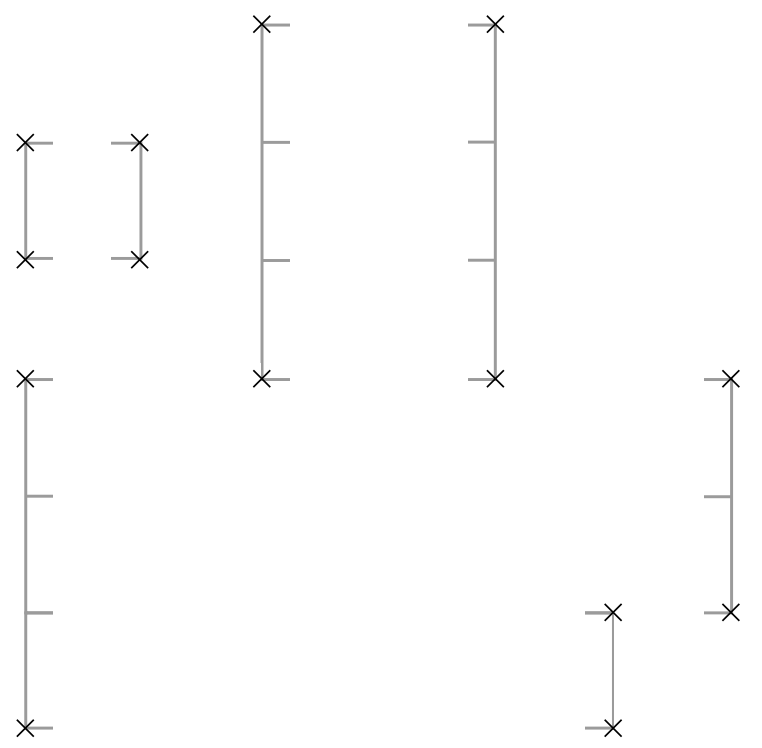

Figure 5: Final vertices of the 2D boundary

by the current stripe, it is not added to the list of $x$ intervals.

Once all edges have been processed, the status data structure contains all the required information to compute the vertices of the boundary.

During the second step, consecutive pairs of stripes are combined using a merge algorithm. The merge algorithm computes the intervalwise xor merge between the stripes. The xor merge is computed by advancing in both lists in parallel. Any vertical $x$ interval that changes from a stripe to the other, that is, they appear in a stripe, but not both, becomes a vertex of the final 2D boundary. This step corresponds to obtaining the vertices (see Figure 5) from the representation computed in step 1 (see Figure 2(b)). 


\subsubsection{Vertex face degree computation}

During the 2D computation, we have to obtain the vertex face degrees in the $x$ and $y$ axes. For the $x$ degrees, we only need to know if the vertical $x$-interval that generated the vertex was facing left or right. For the $y$ degrees, we need to know what side (top or bottom) of the vertex is inside the polygon and which is outside.

Both of these are easily computed, if we make an observation: In each stripe, all x-interval alternate left and right. Moreover, each stripe starts with a left $x$-interval. This allows us to determine directly the $x$ axis degrees. If the vertical $x$-interval was facing left, the $x$ degree will be 0 , and the $-x$ will be 1 . If it was facing right, the $+x$ degree will be 1 , and the $-x$ will be 0 .

For the $y$ degrees, we need to consider the adjacent stripe. It is possible that the vertex is generated by comparison with the upper stripe, or the lower stripe. Additionally, the stripe can fall in the area covered by the adjacent stripe or not. The $y$ degree is computed considering the possible combinations of both mentioned possibilities. If the vertex is obtained by comparing with the upper stripe, the $+y$ degree will be 1 if the $x$-interval is not covered by the upper stripe, $-y$ will be 1 otherwise. Conversely, if it is obtained by comparison with the lower stripe, the $-y$ degree will be 1 if the $x$-interval is not covered by the upper stripe, $+y$ will be 1 otherwise.

\subsubsection{Data structures}

One of the positive characteristics of our method is the simplicity of the data structures it requires.

The status data structure is a vector of horizontal stripes.

A horizontal stripe is a vector of $x$-intervals. It is limited by its two $y$ coordinates, $y$ Min and $y$ Max, and it knows the maximum $x$ coordinate it can reach, $x$ Max. An $x$-interval is a number containing its $x$ coordinate and a boolean to indicate if it's left or right.

We represent a vertex as a $\langle x, y\rangle$ pair, plus the list of $+x,-x,+y,-y$ degrees.

The final boundary is stored in a vector of vertices.

\section{THREE-DIMENSIONAL BOUNDARY EXTRACTION}

\section{Input Set of axis-aligned boxes}

Output Set of vertices that constitute the boundary of the union of the input boxes, and the $x, y$ and $z$ degree of each vertex

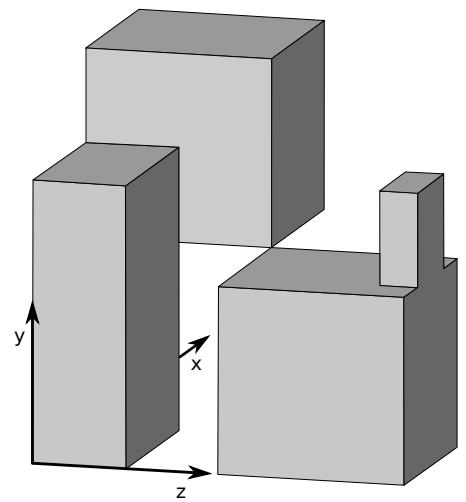

Figure 6: Visualization of the union of a set of four boxes.

The algorithm follows a two step structure. In the first step, the set of boxes is divided into distinct 2D slices, and the 2D boundary of each slice is computed by the method defined in Section 5. In the second step, the obtained 2D boundaries are sequentially compared to obtain the orthogonal polyhedron that constitutes the 3D boundary.

For the first step, we consider a plane perpendicular to the $z$ axis that advances through the boxes. The intersection of the plane with the boxes induces a 2D slice, formed by a set of rectangles. We have to decide in what positions of the plane the 2D slices are computed. The key idea is that a vertex is in the $3 \mathrm{D}$ boundary if it changes between consecutive slices, that is, if the vertex appears in one slice but not in the other. Thus, we consider slices right after each unique $z$ coordinate in the set of boxes. In this manner, the vertices that appear in the boundaries of the slices represent the topology of the 3D boundary at every point in which it changes. As a consequence, local changes between two consecutive slices can be used to obtain the vertices of the 3D boundary. A perspective visualization of a set of boxes (Figure 6) and the slices obtained from it (top part of Figure 7) are shown as an example.

Analogously to the 2D algorithm, separational representation is used for the boxes. They are represented by their two $z$-faces, which we refer to as front if they are oriented towards $+z$, and back if they are oriented towards $-z$. The faces are sorted by $z$, with back faces going before front ones with the same $z$, and the $z$-plane is swept over them. We represent the induced slice as a list of rectangles. To mantain it, when a back face intersects the plane, it is added to the list, and when a front face intersects it, the back face belonging to the same box is removed. After each face is added, we check if the next face in the list has a different $z$. In that case, the 2D boundary of the current state of the slice is computed. Since it is possible 


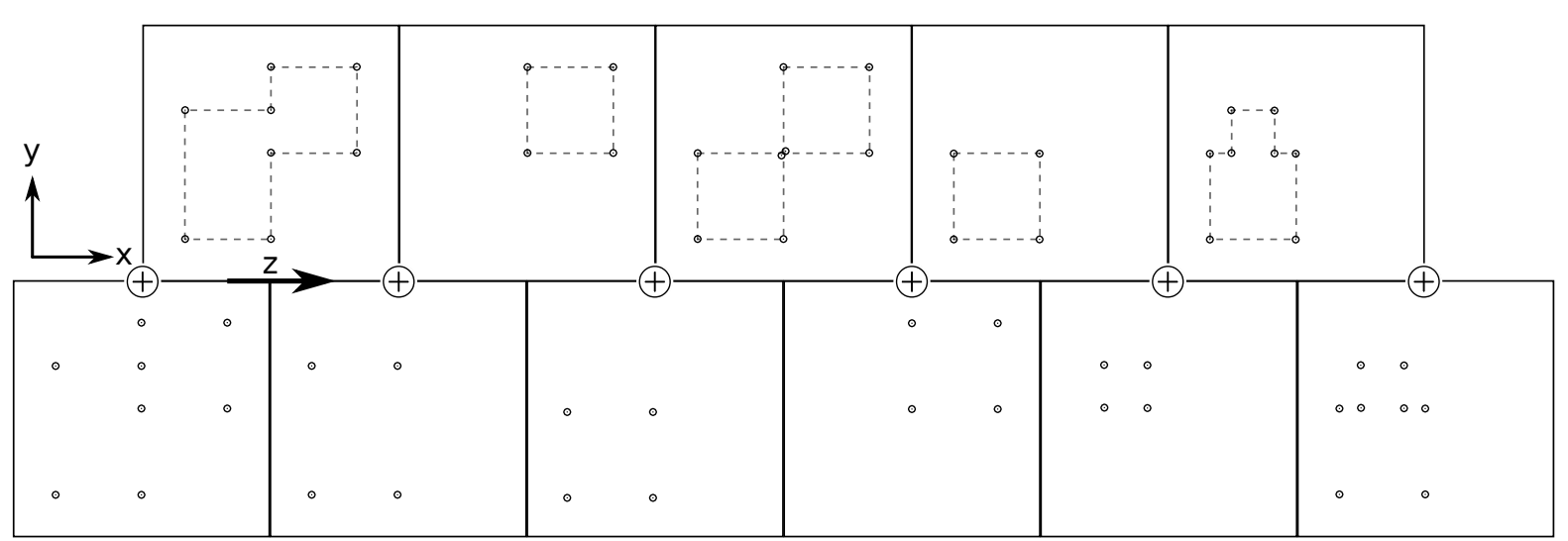

Figure 7: The top part of the figure shows the slices generated from the boxes in Figure 6. The bottom part shows the result of the vertex-wise xor merge. Each of the squares in the bottom corresponds to the xor of the two squares above it.

for a vertex to be generated from the intersection of two faces on the same $z$, one of which is back and the other is front, we also generate the boundary of the slice if the last processed face is back and the next face to process is front. The first step ends when all the faces are processed.

In the second step, the 3D boundary is constructed by a merge algorithm. The vertex-wise xor between each pair of consecutive slices is computed (see Figure 7). All vertices that appear in one slice, but not in both, are in the $3 \mathrm{D}$ boundary. When one such vertex is found, its z-coordinate is set as the previous slice's $z$.

The xor merge is done by advancing in parallel in the two slices. It is necessary to define a strict weak ordering between vertices. The vertices in each slice must be sorted by this ordering. Then, starting by the first vertex on each slice, they are compared. If they are equal, they're not part of the xor, so one element is advanced in both lists. If they are different, we advance an element on the list that had the smaller element, and that element is added as a vertex. This way we can compute which vertices are in one slice but not the other in linear time respect to the number of vertices.

It is worth noting that the algorithm does not require complex data structures. Only basic sequence containers, such as arrays, are required.

The presented algorithm also has the advantage of being independent of the $2 \mathrm{D}$ algorithm chosen, provided the $2 \mathrm{D}$ algorithm generates the necessary output. This output consists only of the vertices and their face degrees in $x$ and $y$, which should be computable straightforwardly.

\subsection{Vertex face degree computation}

During the xor merge, we compute the $z$ face degrees of each vertex. We need the output to be exactly like the one we defined in section 4 , so we have to take a close look at each of the possible configurations. To differentiate them, it is necessary to know where a vertex is, regarding the adjacent boundary.

A 3D vertex can be found in two manners: by comparison with the $2 \mathrm{D}$ boundary in front of it or by comparison with the boundary behind. Independently of that classification, it can also be, regarding the adjacent boundary, inside it or outside it. It is possible that a vertex is exactly in the boundary, and in this case it is classified as either inside or outside and treated accordingly.

If a vertex is outside the adjacent boundary, only the boundary in which the vertex was found has to be considered. Thus, outside front vertices look at $+z$, and outside back vertices at $-z$. Vertices that are inside follow the same reasoning: Only the adjacent boundary is considered for the face degree, so inside front vertices look at $-z$ and inside back vertices at $+z$.

To determine the classification for vertices that are in an edge of the adjacent boundary, we need to look at the already computed $x$ and $y$ face degrees. We have to consider that an edge has only one significant direction, which is $x$ for vertical edges, and $y$ for horizontal ones. If an edge shares its direction with the corresponding direction of the vertex, it means they split the space in the same way. Thus, a vertex that coincides with an edge is classified to be inside if and only if they face the same direction. 


\subsection{Algorithm}

A pseudocode overview of the algorithm is shown (see Algorithm 1). We explain some of the notation used.

The faces have a $z$ coordinate, and the direction they face is indicated. For a given face $f$, these are noted as $f$. $z$ and $f$. front, respectively.

We consider each vertex to be defined by its coordinates and face degrees in the applicable dimensions. For a given vertex $v$, these are noted as $v . x, v . y, v . z$ and v.face_degrees.

We use $\oplus$ to denote the vertex-wise merge xor we defined in Section 6. It should be noted that it computes the $z$ face degree for all resulting vertices.

For brevity, we denote the next element $e$ in a container as next $(e)$.

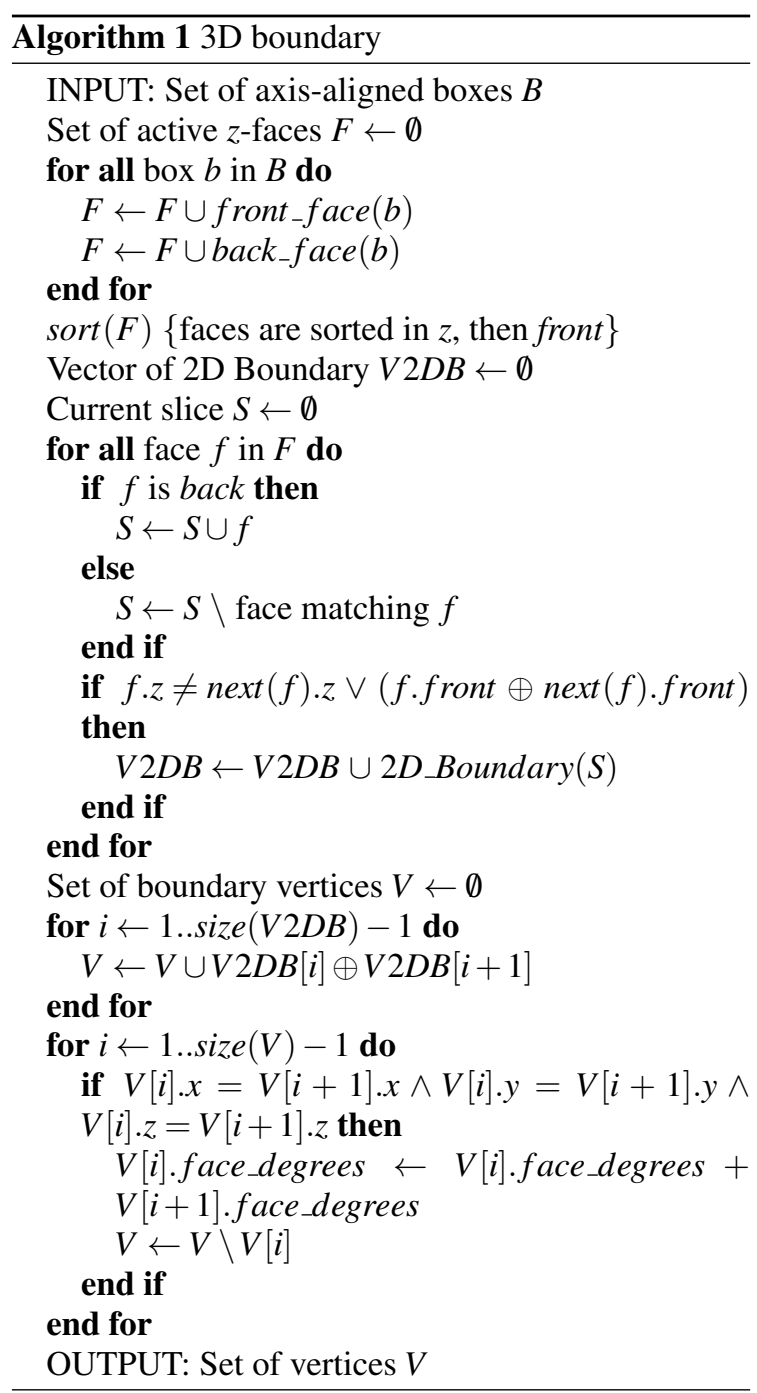

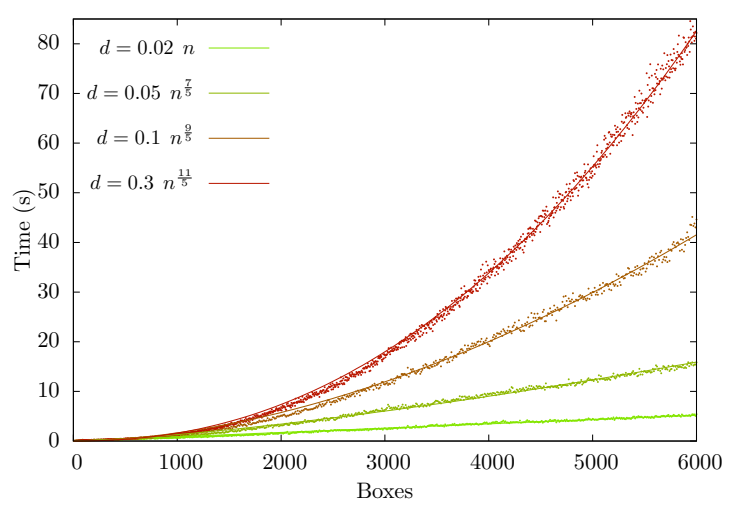

Figure 8: Running times of the presented method to compute the B-rep of several boxes with varying densities. Every point depicts the runtime for a single dataset. Every line corresponds to the curve fitted to average runtime of each density, with respect to the number of boxes.

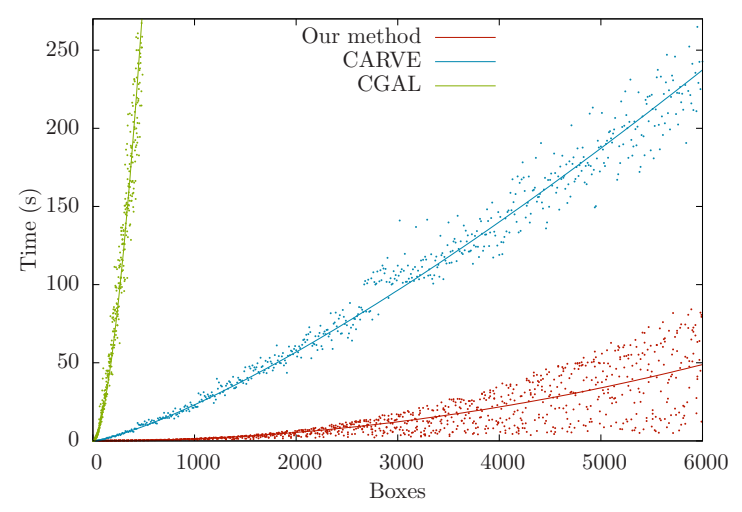

Figure 9: Running times of CARVE to compute the B-rep of several sets of boxes. The plot follows the same scheme of Figure 8.

\section{RESULTS}

The presented method has been implemented in $\mathrm{C}++$. It should be noted that for the two-dimensional boundary computation, Güting's method is not used. A simpler method was implemented for the 2D boundary computation, with a theoretical time complexity of $O\left(n^{2}\right)$, instead of $O(n \log n+k)$. This means that the presented experimental results have room for improvement in the efficiency area. The source code consists of about 800 lines of code, half of them correspond to the two-dimensional boundary computation, and the remaining ones to the threedimensional part. The B-rep extraction is performed using its publically available Python implementation (Vigo, 2011).

All the presented results were performed on PC i7-3770 clocked at $3.9 \mathrm{GHz}$ core and $16 \mathrm{~GB}$ of RAM memory. 
Figure 8 shows the results for over 30000 random cases with varying density of boxes. The density measures the average number of rectangles processed in each slice. The minimum amount, 1 rectangle per slice, corresponds to the value 0 . The maximum amount, $n$ rectangles in each slice, corresponds to 1 . The value increases linearly between both extremes. The formula used is $\frac{n r / 2-n^{2}}{n^{3}-n^{2}}$, where $n$ is the number of boxes and $r$ the total of boxes processed in all slices.

We compared our approach with CGAL and CARVE. We used CGAL's implementation of Nef Polyhedra with an arbitrary precision kernel (Gmpz) (CGAL, 2013). CARVE is a robust octreebased constructive solid geometry library (Sargeant, 2013).

Figure 9 shows a comparison of the running time of our method with the other methods, all with random densities. The computation times of our method include the B-rep computation from the boundary vertices. We observed that the running time of our algorithm is highly correlated with the box density, that is, the average number of rectangles contained in each slice. Thus, it can be seen experimentally that our algorithm is not output-sensitive. It should be noted that none of the randomly generated cases had a density over 0.35 . This implies that inputs approaching the worst case, with densities close to 1 , are extremely rare and do not occur naturally. On average, the runtime performance of the presented approach is of the order $O\left(n^{7 / 5}\right)$, where $n$ is the number of boxes, which coincides with the one obtained by CARVE. CGAL's experimental running time can be fitted to $O\left(n^{9 / 5}\right)$.

Unfortunately, CARVE was unable to terminate the execution for some degenerate inputs, constituting $13 \%$ of the total of datasets. Even for the datasets where it terminated, the generated output was in some instances incorrect. Due to the difficulty of automatically checking the correctness of the CARVE output, we can not estimate the amount those represent. The mentioned errors were detected via manual verification of the output.

\section{CONCLUSION AND FUTURE WORK}

A method was presented to compute the boundary of the union of a set of axis-aligned boxes. It divides the computation of the 3D boundary into a series of $2 \mathrm{D}$ boundary computations, and then combines their results.

As shown in Section 7, the presented method ex-

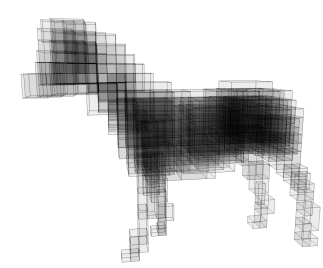

(a)

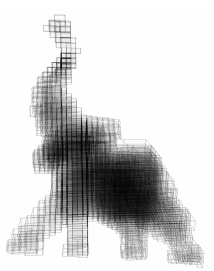

(c)

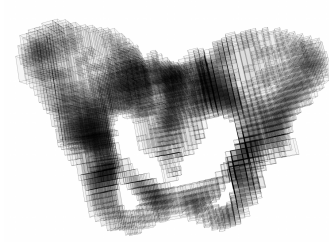

(e) (b)

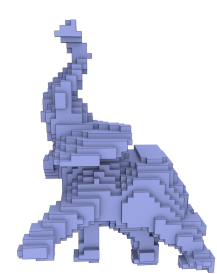

(d)

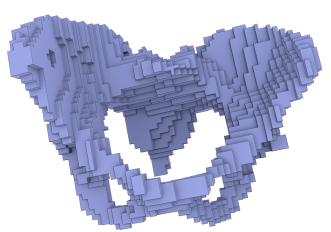

(f)

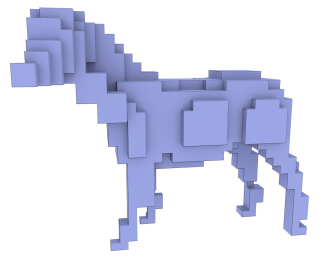

Figure 10: $(a, c, e)$ Set of boxes obtained from voxel models of a horse, an elephant and a human pelvis. Darker shades indicate higher density of boxes. (b, d, f) Visualization of the B-rep obtained with our method each respective input.

hibits lower experimental running time than previous existing general methods, such as CARVE and CGAL (Figure 9). More importantly, it deals with all degenerate cases, generating the correct B-rep in $100 \%$ of the cases. It significantly outperforms CARVE in this regard too, since CARVE failed to terminate in $13 \%$ of cases, and obtained incorrect results in others.

The method is independent from the $2 \mathrm{D}$ implementation, thus any method for computing the $2 \mathrm{D}$ boundary can be used. Interestingly, due to its structure, it allows for parallelization, since in the first step, each call to the 2D boundary method is independent from all others. Furthermore, each xor merge in the second step is also independent, so there is also potential for parallelization in that area.

With the presented method, each 2D boundary is computed independently, when in practice many of the rectangles are shared by adjacent boundaries. This implies that our method potentially performs duplicated computations. The challenge remains to design a dynamic data structure for the boundary of the union of rectangles. Such a data structure would need to keep a representation of the $2 \mathrm{D}$ boundary, and pro- 
vide efficient operations to insert and delete rectangles from it. With it, the duplicated computations that result from treating each 2D slice independently would not be necessary, which would reduce the running time. However, it would lose the independence from the 2D boundary computation, so it would not be easily parallelizable.

The described method only considers the union operation on the set of boxes. However, it would be interesting to consider other boolean operations in which the method could be applied, most notably the intersection. It is possible that our method can be straightforwardly extended to deal with these operations, but careful analysis would be required.

The implementation is available at http:// lafarga.cpl.upc.edu/projectes/boxunion $3 d$ under the GPL license. By doing that, we aim to make it possible for other researchers to use it, as well as contribute to it or modify it.

\section{ACKNOWLEDGEMENTS}

This work has been partially supported by the project TIN2008-02903 and TIN2011-24220 of the Spanish government and by the IBEC (Bioengineering Institute of Catalonia).

\section{REFERENCES}

Aguilera, A. (1998). Orthogonal Polyhedra: Study and Application. PhD thesis, Universitat Politècnica de Catalunya.

Albers, S., Kursawe, K., and Schuierer, S. (1999). Exploring unknown environments with obstacles. In Proceedings of the tenth annual ACM-SIAM symposium on Discrete algorithms, pages 842-843.

Baumann, T., Jans, M., Schömer, E., Schweikert, C., and Wolpert, N. (2008). Dynamic free-space detection for packing algorithms. In EuroCG'08, pages 43-46.

Biedl, T., Durocher, S., and Snoeyink, J. (2009). Reconstructing polygons from scanner data. Lecture Notes in Computer Science, 5878:862-871.

Boissonnat, J.-D., Sharir, M., Tagansky, B., and Yvinec, M. (1998). Voronoi diagrams in higher dimensions under certain polyhedral distance functions. Discrete and Computational Geometry, 19(4):485-519.

Bournez, O., Maler, O., and Pnueli, A. (1999). Orthogonal polyhedra: Representation and computation. Lecture Notes in Computer Science, 1569:46-60.

Campen, M. and Kobbelt, L. (2010). Exact and robust (self-) intersections for polygonal meshes. Computer Graphics Forum, 29:397-406.

CGAL (2013). CGAL, Computational Geometry Algorithms Library. http://www.cgal.org.

Chan, T. M. (2010). A (slightly) faster algorithm for Klee's measure problem. Computational Geometry, 43(3):243-250.

Esperança, C. and Samet, H. (1997). Orthogonal polygons as bounding structures in filter-refine query processing strategies. Lecture Notes in Computer Science, 1262:197-220.

Güting, R. H. (1984). An optimal contour algorithm for isooriented rectangles. Journal of Algorithms, 5(3):303326.

Hachenberger, P. and Kettner, L. (2005). Boolean operations on $3 \mathrm{~d}$ selective nef complexes: optimized implementation and experiments. In Proceedings of the 2005 ACM symposium on Solid and physical modeling, pages $163-174$

Hoffmann, C. (1989). The problems of accuracy and robustness in geometric computation. Computer, 22(3):3139.

Hoffmann, C. (1996). How solid is solid modeling? In Applied Computational Geometry Towards Geometric Engineering, volume 1148 of Lecture Notes in Computer Science, pages 1-8. Springer Berlin Heidelberg.

Jr., W. L. and Preparata, F. P. (1980). Finding the contour of a union of iso-oriented rectangies. Journal of Algorithms, 1(3):235 - 246.

Kaplan, H., Rubin, N., Sharir, M., and Verbin, E. (2007). Counting colors in boxes. In Proceedings of the eighteenth annual ACM-SIAM symposium on Discrete algorithms, pages 785-794.

Klee, V. (1977). Can the measure of $\cup_{n}^{1}\left[a_{i}, b_{i}\right]$ be computed in less than $O(n \log n)$ steps? The American Mathematical Monthly, 84(4):284-285. 
Martinez, J., Pla-Garcia, N., and Vigo, M. (2013). Skeletal representations of orthogonal shapes. Graphical Models, 75:189-207.

Overmars, M. H. and Yap, C.-K. (1991). New upper bounds in Klee's measure problem. SIAM Journal on Computing, 20:1034-1045.

Requicha, A. A. G. and Voelcker, H. (1985). Boolean operations in solid modeling: Boundary evaluation and merging algorithms. Proceedings of the IEEE, 73:3044.

Sargeant, T. (2013). Carve. https://code.google.com/ $\mathrm{p} /$ carve/.

Schifko, M., Jüttler, B., and Kornberger, B. (2010). Industrial application of exact boolean operations for meshes. In Proceedings of the 26th Spring Conference on Computer Graphics, pages 165-172.

Sugihara, K. and Hayashi, Y. (2008). Automatic generation of 3D building models with multiple roofs. Tsinghua Science \& Technology, 13:368 - 374.

Thibault, W. C. and Naylor, B. F. (1987). Set operations on polyhedra using binary space partitioning trees. In Proceedings of the 14th annual conference on Computer graphics and interactive techniques.

Vigo, M. (2011). Orto-brep. http://devel.cpl.upc. edu/orto-brep/.

Vigo, M., Pla, N., Ayala, D., and Martinez, J. (2012). Efficient algorithms for boundary extraction of $2 \mathrm{D}$ and 3D orthogonal pseudomanifolds. Graphical Models, 74(3):61 - 74 .

Wood, D. (1984). The contour problem for rectilinear polygons. Information Processing Letters, 19(5):229-236.

\section{A Usage}

The implementation is available at http:// lafarga.cpl . upc.edu/projectes/boxunion3d under the GPL license.

The software created is divided in two main components: The 3DBoundary program and the B-rep converter.

The process consists on obtaining the boundary vertices using the 3DBoundary program, and then running the B-rep converter to obtain a B-rep of the union of boxes.

\section{A.1 Script}

The script is provided to streamline the process, which performs both steps described below.

Usage:

BoxUnion3D.sh -i INPUTPATH -o OUTPUTPATH [$d-j]$

\section{A.1.1 Input}

The script needs only the path to the input file and the path where output file will be stored.

The input file must be a plain text file, with one box per line. Each line must contain the extreme coordinates of the box in the following format:

$x$ Min $x$ Max yMin yMax zMin zMax

Each of the coordinates must be a real number. Any line not following this will cause a notification and be ignored.

\section{A.1.2 Output}

B-rep reconstruction of the three-dimensional object represented by the input boxes.

\section{A.1.3 Optional parameters}

$-d$ Outputs density file $-j$ Outputs boundary in obj format

\section{A.2 3DBoundary}

Usage:

3DContour INPUTPATH [OPTIONS]

\section{A.2.1 Input}

The INPUTPATH is the path where the input file is located. It must follow the format specified in the above Script section.

\section{A.2.2 Output}

List of vertices of the 3D boundary of the union of input boxes, plus the face degrees of each vertex. Each line contains information about one vertex. The format used is:

$x y z+x+y+z-x-y-z$

Alternatively, the output can be generated in obj format, if the -o option is specified. All the defined information is returned in the standard output.

\section{A.2.3 Optional parameters}

-o outputs a model in obj format

-v outputs information about the computation, useful for debugging purposes

\section{A.3 B-rep converter}

It is an adaptation of a B-rep extractor implemented by Marc Vigo. The data we generate in 3DBoundary makes it unnecesary to perform the full extraction 
from a voxel or evl model. Instead, it computes the B-rep from 3DBoundary's output.

Usage:

python vertexListToBrep.py INPUTPATH OUTPUTPATH

\section{A.3.1 Input}

Same as 3DBoundary output.

\section{A.3.2 Output}

B-rep reconstruction of the three-dimensional object represented by the input vertices. 